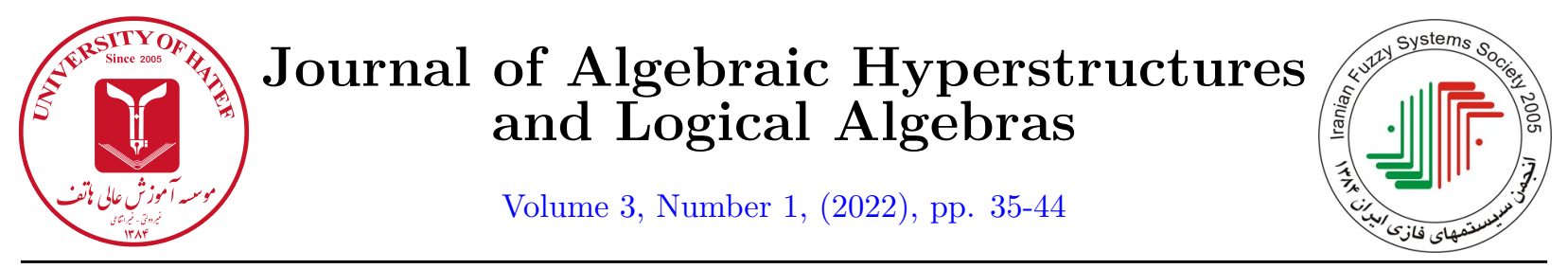

\title{
A short note on categorical equivalences of proper weak pseudo EMV-algebras
}

\author{
A. Dvurečenskij ${ }^{1}$ \\ ${ }^{1}$ Mathematical Institute, Slovak Academy of Sciences, Štefánikova 49, SK-814 73 Bratislava, Slovakia \\ ${ }^{1}$ Palacký University Olomouc, Faculty of Sciences, tr. 17. listopadu 12, CZ-771 46 Olomouc, Czech Republic \\ dvurecen@mat.savba.sk \\ "This paper is dedicated to Professor Antonio Di Nola on the occasion of his 75th birthday."
}

\begin{abstract}
We study the class of weak pseudo EMV-algebras without top element that are a non-commutative generalization of MValgebras, pseudo MV-algebras and generalized Boolean algebras. We present their categorical equivalences to a special category of pseudo MV-algebras with a fixed maximal and normal ideal as well as to a special category of unital $\ell$-groups with a fixed maximal and normal $\ell$-ideal.
\end{abstract}

\section{Article Information}

Corresponding Author:

A. Dvurečenskij;

Received: November 2021;

Accepted: Invited paper;

Paper type: Original.

\section{Keywords:}

Weak pseudo EMV-algebra, pseudo MV-algebra, idempotent element, representation of wPEMV-algebras, proper wPEMV-algebra, associated wPEMV-algebra, categorial equivalence, unital $\ell$-group.

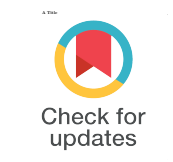

\section{Introduction}

The basic algebraic model for many valued Łukasiewicz logic is an MV-algebra introduced in the late fifties by Chang [3]. During the last decades MV-algebras penetrated into many areas of mathematics as well as they were very deeply studied. The fundamental result on representation of MV-algebras is due to Mundici, [21], saying that the category of MV-algebras is categorically equivalent to the category of unital Abelian $\ell$-groups. For more information about MV-algebras, we recommend to consult with [4]. The importance of MV-algebras and corresponding unital $\ell$-groups entails a whole variety of generalizations of $\mathrm{MV}$-algebras: In [18] and independently in [22], pseudo MV-algebras were introduced as non-commutative MV-algebras. Pseudo BL-algebras as a non-commutative generalization of BL-algebras are defined in [7, 8], and in [19] pseudo hoops were studied, etc. 
Recently, [11, 13, 14] introduced common commutative and non-commutative generalizations of MValgebras, pseudo MV-algebras and generalized Boolean algebras, called EMV-algebras (extended MValgebras) and pseudo EMV-algebras, respectively, as algebras where top element is not assumed. Their basic representation theorem says that they are either equivalent to MV-algebras/pseudo MV-algebras if they possess a top element, or they can be embedded into an equivalent MV-algebra/pseudo MV-algebra as a maximal and normal ideal such that every element outside of the image is a complement of some element from the image. This generalizes the analogous result for generalized Boolean algebra, see [5, Thm 2.2]. For example, the Loomis-Sikorski type theorem for $\sigma$-complete EMV-algebras was established in [12].

We have to underline that not every maximal ideal of an MV-algebra can serve as an example for EMV-algebras.

Since the class of EMV-algebras and pseudo EMV-algebras, respectively, is not a variety, we have studied the least variety containing them and we have showed that this is the class of weak EMV-algebras and the class of weak pseudo EMV-algebras which were introduced and studied in [15] and [16, 17], respectively. The class of wPEMV-algebras is very reach, and the variety of wPEMV-algebras has uncountably many subvarieties whereas the variety of (commutative) wEMV-algebras has only countably many subvarieties. Every wPEMV-algebra admits a representation analogous to the one for pseudo EMV-algebras.

A wPEMV-algebra is not equivalent to a pseudo MV-algebra if and only if it does not admit a top element. Therefore, it is important to study the class of proper wPEMV-algebras, i.e. the class of wPEMValgebras without top element. These structures can be studied also in frames of residuated lattices, see e.g. [1] which cover also semiclans [2] and Bosbach cone algebras [23].

In the paper, we present the categorical equivalence of proper wPEMV-algebras by a special category of pseudo MV-algebras as well as by a special category of unital $\ell$-groups. As corollaries, we present also a subcategories of associated proper ${ }_{\mathrm{w}} \mathrm{PEMV}$-algebras. As a by-product, we obtain categorical equivalences for proper pseudo EMV-algebras.

The paper is organized as follows. Section 2 gives basic facts about studied non-commutative algebras - pseudo MV-algebras, pseudo EMV-algebras, and wPEMV-algebras. The main results, categorical equivalences, are presented in Section 3.

\section{Basic Facts and Notions}

The basic notion for our non-commutative many-valued reasoning is a pseudo MV-algebra defined in [18] (see also an equivalent structure called a generalized MV-algebra [22]). That is, a pseudo $M V$-algebra is an algebra $\left(M ; \oplus,^{-}, \sim, 0,1\right)$ of type $(2,1,1,0,0)$ such that the following axioms hold for all $x, y, z \in M$ with an additional binary operation $\odot$ defined via

$$
y \odot x=\left(x^{-} \oplus y^{-}\right)^{\sim}
$$

(A1) $x \oplus(y \oplus z)=(x \oplus y) \oplus z$;

(A2) $x \oplus 0=0 \oplus x=x$;

(A3) $x \oplus 1=1 \oplus x=1$;

(A4) $1^{\sim}=0 ; 1^{-}=0$;

(A5) $\left(x^{-} \oplus y^{-}\right)^{\sim}=\left(x^{\sim} \oplus y^{\sim}\right)^{-}$;

(A6) $x \oplus\left(x^{\sim} \odot y\right)=y \oplus\left(y^{\sim} \odot x\right)=\left(x \odot y^{-}\right) \oplus y=\left(y \odot x^{-}\right) \oplus x$;

(A7) $x \odot\left(x^{-} \oplus y\right)=\left(x \oplus y^{\sim}\right) \odot y$;

(A8) $\left(x^{-}\right)^{\sim}=x$.

If $\oplus$ is commutative, then a pseudo MV-algebra is an MV-algebra.

Pseudo MV-algebras are intimately connected with unital $\ell$-groups. We note that an element $u$ from the positive cone $G^{+}$of an $\ell$-group $G$ is said to be a strong unit if given $x \in G$, there is an integer $n \geq 0$ 
such that $x \leq n u$. A couple $(G, u)$, where $u$ is a fixed strong unit of $G$, is said to be a unital $\ell$-group. For more info on $\ell$-groups, we recommend to consult [6].

The importance of $\ell$-groups for non-commutative many-valued reasoning is due to the following two facts: (1) If $(G, u)$ is a unital $\ell$-group (not necessarily Abelian), we define

$$
\Gamma(G, u):=[0, u]
$$

and

$$
\begin{aligned}
x \oplus y & :=(x+y) \wedge u, \\
x^{-} & :=u-x, \\
x^{\sim} & :=-x+u, \\
x \odot y & :=(x-u+y) \vee 0,
\end{aligned}
$$

then $\Gamma(G, u):=\left([0, u] ; \oplus,^{-}, \sim, 0, u\right)$ is a pseudo MV-algebra [18].

(2) A basic result on representation of pseudo MV-algebras says that there is a one-to-one correspondence between pseudo MV-algebras and unital $\ell$-groups, for more details see [10], moreover, there is a categorical equivalence between the category of pseudo MV-algebras and the category of unital $\ell$-groups, generalizing a famous result on MV-algebras, see [21].

Pseudo MV-algebras and MV-algebras have many generalizations. One of them are pseudo EMValgebras introduced in $[13,14]$ :

An algebra $(M ; \vee, \wedge, \oplus, 0)$ of type $(2,2,2,0)$ is called a pseudo EMV-algebra (EMV stands for extended $\mathrm{MV}$-algebras) if it satisfies the following conditions:

(E1) $(M ; \vee, \wedge, 0)$ is a distributive lattice with the least element 0 ;

(E2) $(M ; \oplus, 0)$ is an ordered monoid with a neutral element 0 ;

(E3) for each $a \in \mathcal{I}(M):=\{x \in M: x \oplus x=x\}$, (the set of idempotents) the elements

$$
\lambda_{a}(x):=\min \{z \in[0, a]: z \oplus x=a\}, \quad \rho_{a}(x):=\min \{z \in[0, a]: x \oplus z=a\}
$$

exist in $M$ for all $x \in[0, a]$, and the algebra $\left([0, a] ; \oplus, \lambda_{a}, \rho_{a}, 0, a\right)$ is a pseudo MV-algebra;

(E4) for each $x \in M$, there is $a \in \mathcal{I}(M)$ such that $x \leq a$.

If $\oplus$ is commutative, then $\lambda_{a}=\rho_{a}$ and $(M ; \vee, \wedge, \oplus, 0)$ is an EMV-algebra introduced in [11].

In the same way as for pseudo MV-algebras, we can introduce a total binary operation $\odot$ in the following way: For all $x, y \in M$, we define

$$
x \odot y=\rho_{a}\left(\lambda_{a}(y) \oplus \lambda_{a}(x)\right),
$$

where $a \in \mathcal{I}(M)$ and $x, y \in[0, a]$. Then $x \odot y$ is correctly defined and it does not depend on $a \in \mathcal{I}(M)$. It is clear that if $(M ; \oplus,-, \sim, 0,1)$ is a pseudo MV-algebra, then $(M ; \vee, \wedge, \oplus, 0)$ is a pseudo EMV-algebra with top element. Conversely, if $(M ; \vee, \wedge, \oplus, 0)$ is a pseudo EMV-algebra with top element 1 , then $\left(M ; \oplus, \lambda_{1}, \rho_{1}, 0,1\right)$ is a pseudo MV-algebra. This shows that there is a one-to-one correspondence between pseudo MV-algebras and pseudo EMV-algebras with top element.

For example, (1) every generalized Boolean algebra gives a commutative pseudo EMV-algebra.

(2) Given an infinite system $\left\{M_{i}\right\}_{i}$ of pseudo MV-algebras, let $M$ be the system of all sequences $\left(x_{i}\right)_{i} \in$ $\prod_{i} M_{i}$, where $x_{i} \in M_{i}$ and each $x_{i}=0$ for all but finitely many indices $i$. Then $M$ is an example of a pseudo EMV-algebra without top element.

(3) For each pseudo EMV-algebra $M$ without top element, there is a unique pseudo EMV-algebra $N$ with top element such that $M$ can be embedded into $N$ as a maximal and normal ideal of $N$ and every element of $M$ is either in the image of $M$ or is a complement of some element from the image of $M$. This basic representation theorem was established in [14, Thm 6.4].

(4) Not every maximal and normal ideal of a unital $\ell$-group gives an example of a pseudo EMV-algebra. Indeed, take $M=\Gamma(\mathbb{Z} \overrightarrow{\times} G,(1,0))$, where $\mathbb{Z}$ is the set of integers, $\overrightarrow{\times}$ denotes the lexicographic product, 
and $G$ is a non-trivial $\ell$-group. Then $I=\left\{(0, g): g \in G^{+}\right\}$is a unique maximal and normal ideal of $M$, but it cannot serve as an example of a pseudo EMV-algebra in view that it possesses only the zero idempotent element, and it does not dominate any non-zero element of $I$.

For more information about pseudo EMV-algebras consult with [13, 14].

Central algebras of the paper generalizing pseudo MV-algebras and pseudo EMV-algebras are weak pseudo EMV-algebras introduced in [16, 17].

Definition 2.1. An algebra $(M ; \vee, \wedge, \oplus, \ominus, \ominus, 0)$ of type $(2,2,2,2,2,0)$ is called a wPEMV-algebra ( $w$ means weak) if it satisfies the following conditions:

$(W 1)(M, \vee, \wedge, 0)$ is a distributive lattice with the least element 0 ;

(W2) $(M ; \oplus, 0)$ is a monoid;

$(W 3)(y \oplus x) \ominus x \leq y$ and $x \ominus(x \oplus y) \leq y$;

$(W 4)(y \ominus x) \oplus x=x \vee y=x \oplus(x \ominus y)$;

$(W 5) x \ominus(x \wedge y)=x \ominus y$ and $(x \wedge y) \ominus y=x \ominus y$;

$(W 6) y \ominus(x \ominus y)=x \wedge y=(y \ominus x) \ominus y$

$(W 7) z \ominus(x \vee y)=(z \ominus x) \wedge(z \ominus y)$ and $(x \vee y) \ominus z=(x \ominus y) \wedge(y \ominus z)$;

$(W 8)(x \wedge y) \ominus z=(x \ominus z) \wedge(y \ominus z)$ and $z \ominus(x \wedge y)=(z \ominus x) \wedge(z \ominus y)$

(W9) $x \ominus(y \oplus z)=(x \ominus z) \ominus y$ and $(y \oplus z) \ominus x=z \ominus(y \ominus x)$;

$(W 10) x \oplus(y \vee z)=(x \oplus y) \vee(x \oplus z)$ and $(y \vee z) \oplus x=(y \oplus x) \vee(z \oplus x)$

If $\oplus$ is commutative, then $x \ominus y=y \ominus x, x, y \in M$, and the subreduct $(M ; \vee, \wedge, \oplus, \ominus, 0)$ is said to be a weak EMV-algebra which is a commutative algebra introduced in $[15]$.

An element $x \in M$ such that $x \oplus x=x$ is said to be idempotent or Boolean, and let $\mathcal{I}(M)$ be the set of idempotents of $M$. The basic properties of wPEMV-algebras are as follows, see [16, Prop 3.2].

Proposition 2.2. Let $(M ; \vee, \wedge, \oplus, \ominus, \ominus, 0)$ be a wPEMV-algebra and $x, y, z \in M$. Then the following hold:

(i) $(M ; \oplus, 0)$ is an ordered monoid which is right and left naturally ordered (that is, $x \leq y$ if and only if there is $u \in M$ such that $x \oplus u=y$, equivalently, there is $v \in M$ such that $v \oplus x=y$ ).

(ii) $(a \ominus x) \ominus a=x=a \ominus(x \ominus a)$ if $x \leq a$.

(iii) $x \wedge y=((a \ominus x) \vee(a \ominus y)) \ominus a$ and $x \wedge y=a \ominus((x \ominus a) \vee(y \ominus a))$ if $x, y \leq a$.

(iv) $x \leq y$ implies that $x \ominus z \leq y \ominus z$ and $z \ominus x \leq z \ominus y$. Also, $z \ominus y \leq z \ominus x$ and $y \ominus z \leq x \ominus z$.

(v) $z \leq x \oplus y$ if and only if $z \ominus y \leq x$ if and only if $x \ominus z \leq y$.

(vi) $(x \wedge y) \oplus z=(z \oplus z) \wedge(y \oplus z)$ and $z \oplus(x \wedge y)=(z \oplus x) \wedge(z \oplus y)$.

(vii) $z \ominus(x \wedge y)=(z \ominus x) \vee(z \ominus y)$ and $(x \wedge y) \ominus z=(x \ominus z) \vee(y \ominus z)$.

(viii) $x \ominus x=0=x \ominus x$ and $x \ominus 0=x=0 \ominus x$.

(ix) $x \leq y$ if and only if $x \ominus y=0$ if and only if $y \Theta x=0$.

$(\mathrm{x})(x \vee y) \ominus z=(x \ominus z) \vee(y \ominus z)$ and $z \ominus(x \vee y)=(z \ominus x) \vee(z \ominus y)$.

(xi) $x \ominus y \leq x$ and $x \ominus y \leq y$.

(xii) $(x \ominus y) \wedge(y \ominus x)=0=(x \ominus y) \wedge(y \ominus x)$.

(xiii) If $a \oplus a=a$, then $a \oplus x=a \vee x=x \oplus a$. 
(xiv) $z \ominus(x \wedge y)=(z \ominus x) \vee(z \ominus y)$ and $(x \wedge y) \Theta z=(x \ominus z) \vee(y \ominus z)$.

(xv) The binary operation $\oplus$ is commutative if and only if $x \ominus y=y \ominus x$.

We note that every pseudo MV-algebra and every pseudo EMV-algebra can be converted into a weak pseudo EMV-algebra $(M ; \vee, \wedge, \oplus, \ominus, \ominus, 0)$, where

$$
\begin{aligned}
x \ominus y & =x \odot \lambda_{a}(y), \quad \text { where } x, y \leq a \in \mathcal{I}(M), \\
x \ominus y & =\rho_{a}(x) \odot y, \quad \text { where } x, y \leq a \in \mathcal{I}(M) .
\end{aligned}
$$

If $(M ; \vee, \wedge, \oplus, \ominus, \ominus, 0)$ is a wPEMV-algebra such that its reduct $(M ; \vee, \wedge, \oplus, 0)$ is a pseudo EMV-algebra, then the wPEMV-algebra $M$ is said to be an associated wPEMV-algebra.

Finally, let $G^{+}$be the positive cone of an $\ell$-group $G$. Set $x \oplus y=x+y, x \ominus y=(x-y) \vee 0$, and $x \ominus y=(-x+y) \vee 0$ for all $x, y \in G^{+}$. Then $\left(G^{+} ; \vee, \wedge, \oplus, \ominus, \ominus, 0\right)$ is a wPEMV-algebra called a $w P E M V$ algebra of a positive cone or a conic algebra. Then it is cancellative, i.e. if $x \oplus y_{1}=x \oplus y_{2}$, then $y_{1}=y_{2}$ and if $x_{1} \oplus y=x_{2} \oplus y$, then $x_{1}=x_{2}$, and vice versa, every cancellative wPEMV-algebra is isomorphic to some conic algebra, [16, Thm 5.8].

As for pseudo EMV-algebras, we have also a basic representation of wPEMV-algebras by wPEMValgebras with top element:

Theorem 2.3. [Basic Representation Theorem for wPEMV-algebras]

Every wPEMV-algebra $M$ either has a top element and so it is an associated wPEMV-algebra or it can be embedded into an associated wPEMV-algebra $N$ with top element as a maximal and normal ideal of $N$. Moreover, every element of $N$ is either in the image of $x \in M$ or is a right complement of the image of some element $x \in M$.

\section{Categorical equivalences of proper wPEMV-algebras}

A wPEMV-algebra $M$ is said to be proper if $M$ does not contain a top element. We note that the class of proper wPEMV-algebras is not a variety. The class is closed under direct products but neither under subalgebras and nor under homomorphic images. Indeed, take a proper associated wPEMV-algebra $M$ and let $a \in \mathcal{I}(M)$ be non-zero. Then the interval $[0, a]$ is a bounded subalgebra of $M$ and it is a homomorphic image of $M$ under the mapping $f_{a}: M \rightarrow[0, a]$ defined by $f_{a}(x)=x \wedge a, x \in M$. We recall that every proper wPEMV-algebra has infinitely many elements.

We introduce a category PwPEMV such that its objects are proper wPEMV-algebras and morphisms are homomorphisms of wPEMV-algebras. Now, we introduce a special category PPMV of pseudo MValgebras whose objects are couples $(M, I)$, where $M$ is a pseudo MV-algebra and $I$ is a fixed maximal and normal ideal of $M$ such that every element of $M$ belongs either to $I$ or to its complement; we know that $I^{-}:=\left\{x^{-}: x \in I\right\}=\left\{x^{\sim}: x \in I\right\}=: I^{\sim}$. If $\left(M_{1}, I_{1}\right)$ and $\left(M_{2}, I_{2}\right)$ are two objects of PPMV, then a morphism from $\left(M_{1}, I_{1}\right)$ to $\left(M_{2}, I_{2}\right)$ is any homomorphism $\phi: M_{1} \rightarrow M_{2}$ of pseudo MV-algebras such that $\phi\left(I_{1}\right) \subseteq I_{2}$. It is easy to see that PwPEMV and PPMV are categories.

For example, (1) the MV-algebra $M=[0,1]$ has a unique maximal ideal, namely $I=\{0\}$, but $(M, I)$ is not an object of PPMV. Similarly, if $G$ is any $\ell$-group not necessarily Abelian, then $M:=\Gamma(\mathbb{Z} \overrightarrow{\times} G,(1,0))$ is a pseudo MV-algebra (not necessarily an MV-algebra) whose $I:=\left\{(0, g): g \in G^{+}\right\}$is a unique maximal and normal ideal of $M$, so that $(M, I)$ is an object of PPMV.

(2) Let $\mathbb{N}$ be the set of all natural numbers and let $M$ be the Boolean algebra of all finite and co-finite subsets of $\mathbb{N}$. For each $n \in \mathbb{N}$, let $I_{n}=\{A \subset M: n \notin A\}$ and $I_{\infty}=\{A \subset N:|A|<\infty\}$. Then $I_{n}(n \geq 1)$ and $I_{\infty}$ are only maximal ideals of $M$ and each $\left(M, I_{n}\right)$ and $\left(M, I_{\infty}\right)$ are objects of PPMV.

In what follows, we show that both categories are categorically equivalent. The proofs will depend on the following propositions.

Define a mapping $\Phi:$ PPMV $\rightarrow$ PwPEMV as follows: For any object $(M, I) \in$ PPMV, let

$$
\Phi(M, I):=I
$$


and if $\left(M_{1}, I_{1}\right)$ and $\left(M_{2}, I_{2}\right)$ are objects of PPMV and $\phi:\left(M_{1}, I_{1}\right) \rightarrow\left(M_{2}, I_{2}\right)$ is a morphism, then

$$
\Phi(\phi)(x):=\phi(x), \quad x \in I_{1} .
$$

We note that if $(M, I)$ is an object of PPMV, then the restriction of the operations $\vee, \wedge, \oplus, \ominus, \ominus$ from $M$ onto $I$ gives that $I$ is an object of PwPEMV, i.e. $I$ is a proper wPEMV-algebra.

Proposition 3.1. The mapping $\Phi$ is a well-defined functor that is faithful and full from the category PPMV into the category PwPEMV.

Proof. We start with showing that $\Phi$ is a well-defined functor. That is, we show that if $\phi:\left(M_{1}, I_{1}\right) \rightarrow$ $\left(M_{2}, I_{2}\right)$ is a morphism in the category PPMV, then $\Phi(\phi)$ is a morphism in PwPEMV. The mapping $\Phi(\phi)$ is a wPEMV-homomorphism from the proper wPEMV-algebra $I_{1}$ into the proper wPEMV-algebra $I_{2}$.

Let $\phi_{1}$ and $\phi_{2}$ be two morphisms from $\left(M_{1}, I_{1}\right)$ into $\left(M_{2}, I_{2}\right)$ such that $\Phi\left(\phi_{1}\right)=\Phi\left(\phi_{2}\right)$. Then $\phi_{1}(x)=$ $\phi_{2}(x)$ for each $x \in I_{1}$. If $x \in M_{1} \backslash I_{1}$, there is an element $x_{0} \in I_{1}$ such that $x=x_{0}^{-}$. Whence $\phi_{1}(x)=$ $\phi_{1}\left(x_{0}^{-}\right)=\left(\phi_{1}\left(x_{0}\right)\right)^{-}=\left(\phi_{2}\left(x_{0}\right)\right)^{-}=\phi_{2}(x)$ which implies $\phi_{1}=\phi_{2}$, i.e. $\Phi$ is a faithful functor.

Now, we show that $\Phi$ is a full functor, let $h: I_{1} \rightarrow I_{2}$ be a morphism from PPMV, i.e. $h$ is a homomorphism of wPEMV-algebras. By Basic Representation Theorem 2.3 for wPEMV-algebras, there are associated wPEMV-algebras $M_{1}$ and $M_{2}$ with top element such that $I_{1}$ and $I_{2}$ can be embedded into $M_{1}$ and $M_{2}$, respectively, as their maximal and normal ideals. Without loss of generality, we can assume that $I_{i}$ is a wPEMV-subalgebra of $M_{i}$ for $i=1,2$. We assert that there is a morphism $\phi:\left(M_{1}, I_{1}\right) \rightarrow\left(M_{2}, I_{2}\right)$ such that $\Phi(\phi)=h$. That is, $h$ can be extended to a homomorphism $\phi$ of pseudo MV-algebras from $M_{1}$ into $M_{2}$ for some objects $\left(M_{1}, I_{1}\right)$ and $\left(M_{2}, I_{2}\right)$ from PPMV. We set $\phi(x)=h(x)$ and $\phi\left(x^{-}\right)=(h(x))^{-}$if $x \in I_{1}$. Then $\phi(1)=\phi\left(0^{-}\right)=h(0)^{-}=1$. Clearly, $\phi(x)=h(x)$ if $x \in I_{1}$, and $\phi\left(x^{-}\right)=(\phi(x))^{-}, \phi\left(x^{\sim}\right)=h(x)^{\sim}$, $x \in M_{1}$.

We notify that using the Basic Representation Theorem, in each wPEMV-algebra $I$ we can define a binary operation $\odot$ by $x \odot y=\left(y^{\sim} \oplus x^{\sim}\right)^{-}$; equivalently $x \oplus y=\left(y^{-} \oplus x^{-}\right)^{\sim}$ for all $x, y \in I$. We note that $x \odot y \in I$; indeed, if $x \odot y=\left(y^{\sim} \oplus x^{\sim}\right)^{-}=z^{-}$for some $z \in I$, then $y^{\sim}, x^{\sim} \leq y^{\sim} \oplus x^{\sim}=z$ which proves $x^{\sim}, y^{\sim} \in I$, a contradiction. In addition, we have $\phi(x \odot y)=\phi(x) \odot \phi(y)$.

Now let $x, y \in M_{1}$. We show $\phi(x \oplus y)=\phi(x) \oplus \phi(y)$. There are four cases: (1) $x, y \in I_{1}$, then clearly $\phi(x \oplus y)=\phi(x) \oplus \phi(y)$. (2) $x=x_{0}^{-}$and $y=y_{0}^{-}$for some $x_{0}, y_{0} \in I_{1}$. Then $\phi(x \oplus y)=\phi\left(x_{0}^{-} \oplus y_{0}^{-}\right)=$ $\phi\left(\left(y_{0} \odot x_{0}\right)^{-}\right)=\left(\phi\left(y_{0} \odot x_{0}\right)\right)^{-}=\left(\phi\left(y_{0}\right) \odot \phi\left(x_{0}\right)\right)^{-}=\phi\left(x_{0}\right)^{-} \oplus \phi\left(y_{0}\right)^{-}=\phi(x) \oplus \phi(y) .(3) x=x_{0}^{-}$and $y=y_{0}$ for some $x_{0}, y_{0} \in I_{1}$. We get

$$
\begin{aligned}
\phi(x \oplus y) & =\phi\left(x_{0}^{-} \oplus y\right)=\phi\left(\left(y^{\sim} \odot x_{0}\right)^{-}\right)=\phi\left(\left(y \circledast x_{0}\right)^{-}\right) \\
& =\left(\phi(y) \odot \phi\left(x_{0}\right)\right)^{-}=\phi(x) \oplus \phi(y) .
\end{aligned}
$$

(4) $x=x_{0}$ and $y=y_{0}^{-}$. This case is dual to (3).

Summarizing, $\phi$ is a homomorphism of pseudo MV-algebras which is a unique extension of $h$ onto $M_{1}$. Whence, $\Phi(\phi)=h, \phi\left(I_{1}\right) \subseteq I_{2}$, and $\Phi$ is a full functor.

Proposition 3.2. Let $M$ be a proper wPEMV-algebra and $h_{i}: M \rightarrow M_{i}$ be a wPEMV-embedding of $M$ into a wPEMV-algebra $M_{i}$ with top element for $i=1,2$. Define

$$
M_{i}^{0}=\left\{x \in M_{i}: \text { either } x=h_{i}\left(x_{0}\right) \text { or } x=h_{i}\left(x_{0}\right)^{-} \text {for } x_{0} \in M\right\}
$$

for $i=1,2$. Then $\left(M_{1}^{0} ; \oplus,^{-}, \sim, 0,1\right)$ and $\left(M_{2}^{0} ; \oplus,,^{-}, \sim, 0,1\right)$ are isomorphic pseudo $M V$-algebras such that $\left(M_{i}^{0}, h_{i}(M)\right)$ are objects in PPMV for $i=1,2$.

Proof. Let $h_{i}: M \rightarrow N_{i}$ be an embedding for $i=1,2$. Let us define $\phi: N_{1}^{0} \rightarrow N_{2}^{0}$ such that $\phi(x)=h_{2}\left(x_{0}\right)$ if $x=h_{1}\left(x_{0}\right)$ for $x_{0} \in M_{1}$ and $\phi(x)=\left(h_{2}\left(x_{0}\right)\right)^{-}$if $x=h_{1}\left(x_{0}\right)^{-}$for $x_{0} \in M_{1}$. Then, due to the proof of the Proposition 3.1 that $\Phi$ is a full functor, we can prove that $\phi$ is a homomorphism of pseudo MV-algebras. In addition, $\phi$ is a bijection, so that it is an isomorphism. Clearly, $\left(N_{i}^{0}, h_{i}(M)\right) \in$ PPMV for $i=1,2$.

Proposition 3.3. The functor $\Phi$ from the category PPMV into the category PwPEMV has a left-adjoint. 
Proof. We show that for a proper wPEMV-algebra $M$, there is a universal arrow $((N, I), f)$ i.e., $(N, I)$ is an object in PPMV and $f$ is a morphism from $M$ into $\Phi(N, I)=I$ such that if $\left(N^{\prime}, I^{\prime}\right)$ is an object from PwPEMV and $f^{\prime}$ is a morphism from $M$ into $\Phi\left(N^{\prime}, I^{\prime}\right)$, then there exists a unique morphism $f^{*}:(N, I) \rightarrow\left(N^{\prime}, I^{\prime}\right)$ such that $\Phi\left(f^{*}\right) \circ f=f^{\prime}$.

Indeed, by the Basic Representation Theorem 2.3 and Proposition 3.2, there are a unique (up to isomorphism) associated wPEMV-algebra $N$ with top element and an injective wPEMV-homomorphism $f: M \rightarrow N$ such that $f(M)$ is a maximal and normal ideal of $N$ with $f(M) \cup(f(M))^{-}=N$. We assert that $((N, I), f)$ is a universal arrow for $M$. Let $\left(N^{\prime}, I^{\prime}\right)$ be an object from PEMV and let $f^{\prime}$ be a morphism from $M$ into $\Phi\left(N^{\prime}, I^{\prime}\right)$. We can define a mapping $f^{*}: N \rightarrow N^{\prime}$ such that $f^{*}(f(x)):=f^{\prime}(x)$ if $x \in M$ and if $y \in N \backslash f(M)$, there is $y_{0} \in M$ such that $y=\left(f\left(y_{0}\right)\right)^{-}$, and we set $f^{*}(y)=\left(f^{\prime}\left(y_{0}\right)\right)^{-}$. Then $f^{*}: N \rightarrow N^{\prime}$ is a unique pseudo MV-homomorphism such that $\Phi\left(f^{*}\right) \circ f=f^{\prime}$.

Define a mapping $\Psi:$ PwPEMV $\rightarrow$ PPMV by $\Psi(M):=(N, I)$ whenever $((N, I), f)$ is a universal arrow for $M$ and if $f^{\prime}: M \rightarrow M^{\prime}$ is a wPEMV-homomorphism, there is a unique morphism $f^{*}:(N, I) \rightarrow\left(N^{\prime}, I^{\prime}\right)$, where $\Phi\left(N^{\prime}, I^{\prime}\right)=M^{\prime}$, then we set $\Psi\left(f^{\prime}\right):=f^{*}$. Using the Basic Representation Theorem, we have that $\Psi$ is a left-adjoint functor of the functor $\Phi$.

Theorem 3.4. The functor $\Phi$ defines a categorical equivalence of the category PPMV and the category of proper wPEMV-algebras PwPEMV.

In addition, if $h: \Phi(N, I) \rightarrow \Phi\left(N^{\prime}, I^{\prime}\right)$ is a morphism of proper wPEMV-algebras, then there is a unique homomorphism $\phi: N \rightarrow N^{\prime}$ of pseudo $M V$-algebras with $\phi(I) \subseteq I^{\prime}$ such that $h=\Psi(\phi)$, and

(i) if $h$ is surjective, so is $\phi$;

(ii) if $h$ is injective, so is $\phi$.

Proof. According to [20, Thm IV.4.1 (i),(iii)], since $\Phi$ is faithful and full, by Proposition 3.1, and it has a left-adjoint, by Proposition 3.3, then it is necessary to show that, for any proper wPEMV-algebra $M$, there is an object $(N, I)$ in PPMV such that $\Phi(N, I)$ is isomorphic to $M$. For this, it is sufficient to take any universal arrow $((N, I), f)$ of $M$.

We introduce two subcategories of PwPEMV and of PPMV, respectively, corresponding to associated proper wPEMV-algebras. Thus, let $\mathrm{PwPEMV}_{a}$ be the set of associated proper wPEMV-algebras; it is equivalent to the set of proper pseudo EMV-algebras. In addition, let PwPEMV ${ }_{a}$ be the set of $(N, I) \in \operatorname{PwPEMV}$ such that $I$ is a maximal and normal ideal of the pseudo MV-algebra $N$ having enough idempotents, i.e. given $x \in I$, there is an idempotent $a \in I$ such that $x \leq a$. Then $\mathrm{PwPEMV}_{a}$ and $\mathrm{PPMV}_{a}$ are proper subcategories of PwPEMV and PPMV, respectively. Theorem 3.4 entails the following categorical equivalence.

Corollary 3.5. The subcategories $\mathrm{PwPEMV}_{a}$ and $\mathrm{PPMV}_{a}$ are categorically equivalent.

Proof. Let $G$ be a non-zero $\ell$-group and define the pseudo MV-algebra $N=\Gamma(\mathbb{Z} \overrightarrow{\times} G,(1,0))$ with the

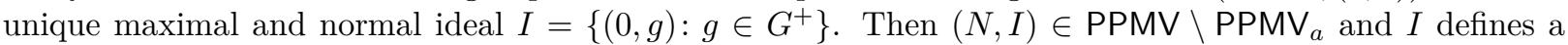
non-associated proper wPEMV-algebra. If $\Phi_{a}$ and $\Psi_{a}$ denote the restrictions of $\Phi$ and $\Psi$ onto PPMV and PwPEMV, respectively, then they are functors, and by Theorem 3.4, $\Phi_{a}$ defines a categorical equivalence in question.

We note that the latter corollary gives also a categorical equivalence for proper pseudo EMV-algebras.

We introduce the following partial addition + on every pseudo MV-algebra $N: x+y$ is defined iff $y \odot x=0$, and in such a case, $x+y:=x \oplus y$; if $N=\Gamma(G, u)$, then + coincides with the group addition restricted to $[0, u]$. We say that a couple $(G, f)$ is a universal group for a pseudo MV-algebra $N$ if (i) $f$ is a mapping from $N$ into a po-group $G$ which preserves partial addition + on $N$ such that $G=G^{+}-G^{+}$, $f(N)$ generates $G^{+}$as a semigroup, (ii) for any group $K$ and any +-preserving mapping $h: N \rightarrow K$, there is a group homomorphism $\phi: G \rightarrow K$ such that $h=\phi \circ f$. Due to [10, Thm 5.3], if $N \cong \Gamma(G, u)$, then $(G, f)$ is a universal group for $N$, where $f$ is an isomorphism $f: N \rightarrow \vec{\Gamma}(G, u)$.

Now, we define a special category MNLUG of unital $\ell$-groups whose objects are triples $(G, u, I)$, where $(G, u)$ is a unital $\ell$-group and $I$ is a fixed maximal and normal $\ell$-ideal of $G$ such that the $\ell$-group generated by $I$ and $u$ is $G$. A morphism $f:\left(G_{1}, u_{1}, I_{1}\right) \rightarrow\left(G_{2}, u_{2}, I_{2}\right)$ is any homomorphism of unital $\ell$-groups $f: G_{1} \rightarrow G_{2}$ such that $f\left(I_{1}\right) \subseteq I_{2}$. 
Let us define a functor $\Gamma_{I}:$ MNLUG $\rightarrow$ PPMV as follows: If $(G, u, I)$ is an object of MNLUG, then

$$
\Gamma_{I}(G, u, I):=(\Gamma(G, u), I \cap[0, u]),
$$

and if $f$ is a morphism from an object $\left(G_{1}, u_{1}, I_{1}\right)$ into another object $\left(G_{2}, u_{2}, I_{2}\right)$, then

$$
\Gamma_{I}(f)(x):=f(x), \quad x \in \Gamma(G, u) .
$$

Proposition 3.6. $\Gamma_{I}$ is a well-defined functor that is faithful and full. Moreover, it has a left-adjoint.

Proof. Clearly $\Gamma_{I}(G, u, I)=(\Gamma(G, u), I \cap[0, u]) \in$ PPMV. If $f:\left(G_{1}, u_{1}, I_{1}\right) \rightarrow\left(G_{2}, u_{2}, I_{2}\right)$ is a morphism, then the restriction of $f$ onto $\Gamma\left(G_{1}, u_{1}\right)$ is in fact a homomorphism of pseudo MV-algebras with $f\left(I_{1}\right) \subseteq I_{2}$, so that $\Gamma_{I}(f)\left(I_{1} \cap\left[0, u_{1}\right]\right) \subseteq I_{2} \cap\left[0, u_{2}\right]$, and, $\Gamma_{I}$ is a correctly defined functor.

Let $f_{1}$ and $f_{2}$ be two morphisms from $\left(G_{1}, u_{1}, I_{1}\right)$ into $\left(G_{2}, u_{2}, I_{2}\right)$ such that $\Gamma_{I}\left(f_{1}\right)=\Gamma_{I}\left(f_{2}\right)$. Then $f_{1}(x)=f_{2}(x)$ for each $x \in \Gamma\left(G_{1}, u_{1}\right)$, so that $f_{1}(x)=f_{2}(x)$ for each $x \in G_{1}$ and $f_{1}=f_{2}$.

Now, let $\phi: \Gamma_{I}\left(G_{1}, u_{1}, I_{1}\right)=\left(\Gamma\left(G_{1}, u_{1}\right), I_{1} \cap\left[0, u_{1}\right]\right) \rightarrow \Gamma_{I}\left(G_{2}, u_{2}, I_{2}\right)=\left(\Gamma\left(G_{2}, u_{2}\right), I_{2} \cap\left[0, u_{2}\right]\right)$ be a morphism. According to the proof of [10, Prop 6.1], we can uniquely extend $\phi$ to a homomorphism of unital $\ell$-groups $f: G_{1} \rightarrow G_{2}$. Applying [9, Thm 6.1], $I_{i} \cap\left[0, u_{i}\right]$ can be uniquely extended to the $\ell$-ideal $I_{i}, i=1,2$, we have $f\left(I_{1}\right) \subseteq I_{2}$ and $f$ is a morphism from $\left(G_{1}, u_{1}, I_{1}\right)$ into $\left(G_{2}, u_{2}, I_{2}\right)$. Finally, we have established $\Gamma_{I}$ is a full functor because $\Gamma_{I}(f)=\phi$.

To show that $\Phi_{I}$ has a left-adjoint, we show that for an object $\left(N, I_{0}\right)$ in PPMV, there is a universal arrow $((G, u, I), f)$, i.e. $(G, u, I)$ is an object from MNLUG and $f$ is a morphism from $\left(N, I_{0}\right)$ into $\Gamma_{I}(G, u, I)=$ $(\Gamma(G, u), I \cap[0, u])$ such that if $\left(G^{\prime}, u^{\prime}, I^{\prime}\right)$ is an object from MNLUG and $f^{\prime}$ is a morphism from $\left(N, I_{0}\right)$ into $\Gamma_{I}\left(G^{\prime}, u^{\prime}, I^{\prime}\right)=\left(\Gamma\left(G^{\prime}, u^{\prime}\right), I^{\prime} \cap\left[0, u^{\prime}\right]\right)$, then there is a unique morphism $f^{*}:(G, u, I) \rightarrow\left(G^{\prime}, u, I^{\prime}\right)$ such that $\Gamma_{I}\left(f^{*}\right) \circ f=f^{\prime}$.

Take the universal group $(G, f)$ for the pseudo MV-algebra $N$. Then $f$ is a pseudo MV-bijection from $N$ onto $\Gamma(G, u)$. We assert that $((G, u, I), f)$ is a universal arrow for $\left(N, I_{0}\right)$, where $I$ is an $\ell$-ideal of $G$ generated by $f\left(I_{0}\right)$. Indeed, take an object $\left(G^{\prime}, u^{\prime}, I^{\prime}\right)$ from MNLUG and let $f^{\prime}$ be a morphism from $\left(N, I_{0}\right) \rightarrow \Gamma_{I}\left(G^{\prime}, u^{\prime}, I^{\prime}\right)=\left(\Gamma\left(G^{\prime}, u^{\prime}\right), I_{0}^{\prime}\right)$, where $I_{0}^{\prime}=I^{\prime} \cap\left[0, u^{\prime}\right]$. Since $f: M \rightarrow \Gamma(G, u) \subset G^{+}$is a +preserving mapping and $f^{\prime}: M \rightarrow \Gamma\left(G^{\prime}, u^{\prime}\right) \subseteq G^{\prime}$ is also a +-preserving mapping, then there is a unique homomorphism of unital $\ell$-groups $f^{*}: G \rightarrow G^{\prime}$ such that $f^{*} \circ f=f^{\prime}$. First, if $x \in I_{0}$, then $f(x) \in I$ and thus $f^{*}(f(x))=f^{\prime}(x) \in I_{0}^{\prime}$.

We recall that if $I_{0}$ is an ideal of $\Gamma(G, u)$, then the $\ell$-ideal $I$ of $G$ generated by an ideal $I_{0}$ of $\Gamma(G, u)$ is $I=\left\{x \in G: \exists x_{i}, y_{j} \in I_{0}, x=x_{1}+\cdots+x_{n}-y_{1}-\cdots-y_{m}\right\}$.

Therefore, if $x \in I$ is arbitrary, $f^{\prime}(x) \in I^{\prime}$, so that $f^{*}$ is also a morphism from $(G, u, I)$ to $\left(G^{\prime}, u, I^{\prime}\right)$, i.e. $((G, u, I), f)$ is the required universal arrow.

Define a mapping $\Xi_{I}:$ PPMV $\rightarrow$ MNLUG by $\Xi_{I}\left(N, I_{0}\right)=(G, u, I)$ if $((G, u, I), f)$ is a universal arrow for $\left(N, I_{0}\right)$ and $I$ is a maximal $\ell$-ideal of $G$ generated by $f\left(I_{0}\right)$. If $f^{\prime}$ is a morphism from $\left(N, I_{0}\right)$ into $\left(N^{\prime}, I_{0}^{\prime}\right)$, there is a unique morphism $f^{*}:(G, u, I) \rightarrow\left(G^{\prime}, u^{\prime}, I^{\prime}\right)$, where $N^{\prime} \cong \Gamma\left(G^{\prime}, u^{\prime}\right)$ and $I^{\prime}$ is a maximal $\ell$-ideal of $G^{\prime}$ generated by $f^{\prime}\left(I_{0}^{\prime}\right)$, consequently $\Xi_{I}\left(f^{\prime}\right):=f^{*}$. Therefore, $\Xi_{I}$ is a left-adjoint of $\Gamma_{I}$.

The last results entail the following categorical equivalence:

Theorem 3.7. The categories MNLUG, PPMV and PwPEMV are mutually categorically equivalent.

Proof. The categorical equivalence of PPMV and PwPEMV follows from Theorem 3.4, and using ideas analogous to the proof of Theorem 3.4, we can show that MNLUG and PPMV are categorical equivalent, too.

Finally, let $\mathrm{MNLUG}_{a}$ be the set of objects $(G, u, I) \in$ MNLUG such that, for each $x \in I \cap[0, u]$, there is an idempotent $a \in I \cap[0, u]$ with $x \leq a$. Then MNLUG $_{a}$ is a proper subcategory of MNLUG $_{a}$ and using Corollary 3.5 and ideas from proof of Corollary 3.5, we can establish the next corollary.

Corollary 3.8. The categories $\mathrm{PvPEMV}_{a}, \mathrm{PPMV}_{a}$ and $\mathrm{MNLUG}_{a}$ are mutually categorical equivalent.

We note that the latter result gives also a categorical equivalences for the set of proper pseudo EMValgebras because every proper associated wPEMV-algebra is in fact a proper pseudo EMV-algebra. 


\section{Conclusion}

The class of proper weak pseudo EMV-algebras is the class of weak pseudo EMV-algebras that are not equivalent to pseudo MV-algebras. Therefore, we characterize the categorical equivalence of the category of proper wPEMV-algebras by a special category of pseudo MV-algebras as couples consisting of a pseudo MV-algebra and a fixed maximal and normal ideal such that every element of the pseudo MV-algebra belongs either to the ideal or is a complement of some element from the ideal, Theorem 3.4. An analogous result is given by a special class of unital $\ell$-groups with a fixed maximal and normal $\ell$-ideal, see Theorem 3.7. In addition, we characterize also a subcategory of associated proper wPEMV-algebras and we found for them categorical representations, see Corollary 3.5 and Corollary 3.8. As a by-product, we have obtained categorical equivalences for proper pseudo EMV-algebras.

\section{Acknowledgment}

The author acknowledges the support by the Slovak Research and Development Agency under contract APVV-20-0069 and the grant VEGA No. 2/0142/20 SAV

\section{References}

[1] P. Bahls, J. Cole, N. Galatos, P. Jipsen, C. Tsinakis, Cancellative residuated lattices, Algebra Universalis, 50 (2003), 83-106.

[2] B. Bosbach, Concerning semiclans, Archiv der Mathematik, 37 (1981), 316-324.

[3] C.C. Chang, Algebraic analysis of many-valued logics, Transactions of the American Mathematical Society, 88 (1958), 467-490.

[4] R. Cignoli, I.M.L. D’Ottaviano, D. Mundici, Algebraic foundations of many-valued reasoning, Springer Science and Business Media, Dordrecht, 2000.

[5] P. Conrad, M.R. Darnel, Generalized Boolean algebras in lattice-ordered groups, Order, 14 (1998), $295-319$.

[6] M.R. Darnel, Theory of lattice-ordered groups, Marcek Dekker, Inc., New York, Basel, Hong Kong, 1995.

[7] A. Di Nola, G. Georgescu, A. Iorgulescu, Pseudo-BL-algebras: Part I, Multi-Valued Logic, 8 (2002), $673-714$

[8] A. Di Nola, G. Georgescu, A. Iorgulescu, Pseudo-BL-algebras: Part II, Multi-Valued Logic, 8 (2002), $715-750$.

[9] A. Dvurečenskij, States on pseudo MV-algebras, Studia Logica, 68 (2001), 301-327.

[10] A. Dvurečenskij, Pseudo MV-algebras are intervals in $\ell$-groups, Journal of the Australian Mathematical Society, 72 (2002), 427-445.

[11] A. Dvurečenskij, O. Zahiri, On EMV-algebras, Fuzzy Sets and Systems, 373 (2019), 116-148.

[12] A. Dvurečenskij, O. Zahiri, The Loomis-Sikorski theorem for EMV-algebras, Journal of the Australian Mathematical Society, 106 (2019), 200-234.

[13] A. Dvurečenskij, O. Zahiri, Pseudo EMV-algebras. I. Basic properties, Journal of Applied LogicsIFCoLog Journal of Logics and their Applications, 6 (2019), 1285-1327.

[14] A. Dvurečenskij, O. Zahiri, Pseudo EMV-algebras. II. Representation and states, Journal of Applied Logics-IFCoLog Journal of Logics and their Applications, 6 (2019), 1329-1372. 
[15] A. Dvurečenskij, O. Zahiri, A variety containing EMV-algebras and Pierce sheaves of EMV-algebras, Fuzzy Sets and Systems, 418 (2021), 101-125, Doi:10.1016/j.fss.2020.09.011.

[16] A. Dvurečenskij, O. Zahiri, Weak pseudo EMV-algebras. I. Basic properties, Journal of Applied LogicIfCoLog Journal of Logics and their Applications, 8 (2021), 2365-2399.

[17] A. Dvurečenskij, O. Zahiri, Weak pseudo EMV-algebras. II. Representation and subvarieties, Journal of Applied Logic - IfCoLog Journal of Logics and their Applications, 8 (2021), 2401-2433.

[18] G. Georgescu, A. Iorgulescu, Pseudo MV-algebras, Multiple-Valued Logics, 6 (2001), 193-215.

[19] G. Georgescu, L. Leuştean, V. Preoteasa, Pseudo-hoops, Journal of Multiple-Valued Logic and Soft Computing, 11 (2005), 153-184.

[20] S. Mac Lane, Categories for the working mathematician, Springer-Verlag, New York, Heidelberg, Berlin, 1971.

[21] D. Mundici, Interpretation of AF $C^{*}$-algebras in Eukasiewicz sentential calculus, Journal of Functional Analysis, 65 (1986), 15-63.

[22] J. Rachůnek, A non-commutative generalization of MV-algebras, Czechoslovak Mathematical Journal, 52 (2002), 255-273.

[23] W. Rump, Y. Yang, A note on Bosbach's cone algebras, Studia Logica, 98 (2011), 375-386. 